\title{
MPMI ACKNOWLEDGMENT OF REVIEWERS
}

The success of Molecular Plant-Microbe Interactions depends on the quality of manuscripts submitted by authors and on the care and competence with which they are reviewed. It is the policy of the Editorial Board to solicit reviews of manuscripts from specialists most qualified to review them. In addition to members of the Editorial Board, the individuals listed below provided constructive critical reviews of one or more manuscripts during the past year. Their names are published here in grateful appreciation for their contributions to the journal.

G. Adam, BOKU, University of Natural Resources and Applied Life Sciences, Vienna, Austria

J. R. Alfano, University of Nebraska, Lincoln, NE, U.S.A. A., Alvarez, University of Hawaii, Honolulu, HI, U.S.A. A. Anand, Sam Roberts Noble Foundation, Ardmore, OK, U.S.A.

P. A. Anderson, Flinders University, Adelaide, South Australia, Australia

M. Arlat, INRA-CNRS, Castanet Tolosan, France

F. O. Asiegbu, Swedish University of Agricultural Sciences, Uppsala, Sweden

F. M. Ausubel, Massachusetts General Hospital, Boston, MA, U.S.A.

M. Bailey, Institute of Virology and Environmental Microbiology, Oxford, U.K.

B. Baker, University of California Berkeley, Albany, CA, U.S.A.

S. E. Baker, Pacific Northwest National Laboratory, Richland, WA, U.S.A.

I. Barash, Tel-Aviv University, Tel-Aviv, Israel

C. Baron, McMaster University, Hamilton, Ontario, Canada

D. Baulcombe, The Sainsbury Laboratory, John Innes

Centre, Colney Lane, Norwich, U.K.

T. Baum, Iowa State University, Ames, IA, U.S.A.

S. Beer, Cornell University, Ithaca, New York, U.S.A.

A. Bent, University of Wisconsin-Madison, Madison, WI, U.S.A.

C. Beveridge, University of Queensland, Brisbane, Queensland, Australia

S. Bieri, University of Zürich, Zürich, Switzerland

P. Birch, SCRI, Dundee, U.S.A.

D. McK. Bird, North Carolina State University, Raleigh,

NC, U.S.A.

T. Bisseling, Wageningen University, Wageningen, The Netherlands

J. Boch, Martin Luther University, Halle (Saale), Germany

J. Boddu, University of Minnesota, St Paul, MN, U.S.A.

A. Bogdanove, Iowa State University, Ames, IA, U.S.A.

L. Borge, Royal Holloway, University of London, Egham, U.K.

R. M. Bostock, University of California, Davis, CA, U.S.A.

N. J. Brewin, John Innes Centre, Norwich, U.K.

W. Broughton, University of Geneva, Geneva, Switzerland

D. W. Brown, USDA-ARS-NCAUR, Peoria, IL, U.S.A.

I. Brunner, WSL, Birmensdorf, Switzerland

G. Bryan, Scottish Crop Research Institute, Dundee, U.K.

M. Bucher, ETH Zurich, Lindau, Switzerland

T. J. Burr, Cornell University, Geneva, NY, U.S.A.

K. Caldwell, University of California, Davis, CA, U.S.A.

R. Cameron, McMaster University, Hamilton, Canada

R. Carlson, University of Georgia, Athens, GA, U.S.A.

J. P. Carr, Cambridge University, Cambridge, U.K.

B. Carroll, The University of Queensland, Brisbane,

Australia

P. Castagnone Sereno, INRA, Sophia Antipolis, France
M. Chalot, UHP Nancy I, Vandoeuvre, France

A. Charkowski, University of Wisconsin, Madison, WI, U.S.A.

T. Charles, University of Waterloo, West Waterloo, ON, Canada

A. Chatterjee, University of Missouri, Columbia, MO, U.S.A.

Z. Chen, Purdue University, West Lafayette, In, U.S.A.

M. Chiurazzi, Consiglio Nazionale delle Ricerche (CNR),

Napoli, Italy

P. Christie, University of Texas -Houston Medical School,

Houston, TX, U.S.A.

K.-R. Chung, University of Florida, Lake Alfred, FL, U.S.A.

A. Churchill, Cornell University, Ithaca, NY, U.S.A.

S. J. Clough, USDA-ARS, Urbana, IL, U.S.A.

A. Collmer, Cornell University, Ithaca, NY, U.S.A

D. R. Cook, University of California, Davis, CA, U.S.A.

J. Cooper, The Queens University of Belfast, Belfast, U.K.

D. Coplin, Ohio State University, Columbus, OH, U.S.A.

P. Coutos-Thévenot, Faculté des Scienes de Poitiers,

Poitiers, France

V. Crane, Pioneer Hi-Bred, Johnston, IA, U.S.A.

M. Crespi, CNRS, Gif Sur Yvette, France

J. N. Culver, University of Maryland Biotechnology

Institute, College Park, MD, U.S.A.

M. J. Davis, University of Florida, Homestead, FL, U.S.A.

E. Davis, North Carolina State University, Raleigh, NC,

U.S.A.

W. Dawson, University of Florida, Winter Haven, FL,

U.S.A.

Italy

G. De Lorenzo, Universitá di Roma La Sapienza, Roma,

P. J.G.M. De Wit, Wageningen University, Wageningen, The

Netherlands

W. J. Deakin, University of Geneva, Geneva, Switzerland

F. Debelle, LIPM INRA-CNRS, Castanet-Tolosan, France

H. Bruno, Deising, Martin-Luther University Halle, Halle

(Saale), Germany

T. P. Delaney, The University of Vermont, Burlington, VT,

U.S.A.

M. Delledonne, Universitá degli Studi di Verona, Verona, Italy

C. M. Deom, University of Georgia, Athens, GA, U.S.A.

Y. Dessaux, Institut des Sciences du Vegetal, Gif-sur-Yvette, France

A. Di Pietro, Universidad de Cordoba, Cordoba, Spain

M. Dickman, University of Nebraska, Lincoln, NE, U.S.A.

M. A. Djordjevic, Australian National University, Canberra,

ACT, Australia

V. Dolja, Oregon State University, Corvallis, OR, U.S.A.

N. M. Donofrio, North Carolina State University, Raleigh,

NC, U.S.A.

J. M. Dow, University College Cork, Cork, Ireland

A. Downie, John Innes Centre, Norwich, U.K.

I. Dry, CSIRO PI, Glen Osmond, South Australia, Australia

R. Dudler, University of Zurich, Zurich, Switzerland 
C. Dunand, University of Geneva, Genève, Switzerland

K. E. Duncan, DuPont, Wilmington, DE, U.S.A.

L. Dunkle, Purdue University, West Lafayette, IN, U.S.A.

J. Durner, GSF, Munich/Neuherberg, Germany

J. Duvick, Pioneer Hi-Bred, International, Inc., Johnston,

IA, U.S.A.

D. J. Ebbole, Texas A \& M University, College Station, TX, U.S.A.

T. H. N. Ellis, John Innes Centre, Norwich, U.K.

C. Elmerich, Inst Des Sciences Du Vegetal, Gif-sur-Yvette, France

D. Emerich, Univeristy of Missouri, Columbia, MO, U.S.A.

G. Espin, Instituto de Biotecnologia UNAM, Cuernavaca,

Morelos, Mexico

T. Eulgem, University of California, Riverside, CA, U.S.A.

D. Expert, CNRS ISV, Paris, CEDEX 05, France

C. Farah, University São Paulo, São Paulo, SP, Brazil

M. Farman, University of Kentucky, Lexington, KY, U.S.A

S. K. Farrand, University of Illinois at Urbana-Champaign, Urbana, IL, U.S.A.

G. P. Ferguson, Edinburgh University, Edinburgh, U.K.

R. D. Finlay, SLU, Uppsala, Sweden

H. M. Fischer, Inst Fur Mikrobiologie ETH, Zürich, Switzerland

E. Flemetakis, Agricultural University of Athens, Athens, Greece

J. Fletcher, Oklahoma State University, Stillwater, OK, U.S.A.

V. Flors, University Jaume I, Castellon, Spain

P. R. Fobert, Plant Biotechnology Institute, Saskatoon,

Saskatchewan, Canada

R. G. Fray, University of Nottingham, Leicestershire, U.K.

P. Gamas, INRA-CNRS, Castanet Tolosan cedex, France

F. Garcia-Arenal, Univ Politecnica De Madrid, Madrid, Spain

W. Gassmann, University of Missouri-Columbia, Columbia,

MO, U.S.A.

C. Gebhardt, Mac-Planck-Institute for Plant Breeding

Research, Cologne, Germany

K. Geider, BBA Dossenheim, Dossenheim, Germany

S. Genin, CNRS-INRA, Castanet Tolosan, France

S. R. Giddens, University of Oxford Oxford, Oxon, U.K.

R. Gilbertson, University of California, Davis, CA, U.S.A.

J. Glazebrook, University of Minnesota, St. Paul, MN,

U.S.A.

A. Glenn, USDA, ARS, Russell Research Center, Athens,

GA, U.S.A.

B. Glick, University of Waterloo, Waterloo, Ontario, Canada

F. Goggin, University of Arkansas, Fayetteville, AR, U.S.A.

R.W. Goldbach, Wageningen University, Wageningen, The

Netherlands

J. E. Gonzalez, University of Texas, Richardson, TX, U.S.A.

J. Green, University Birmingham, Birmingham, U.K.

J. Greenberg, University of Chicago, Chicago, IL, U.S.A.

P. M. Gresshoff, University of Queensland, Brisbane,

Queensland, Australia

D. Gross, Texas A\&M University, College Station, TX, U.S.A.

R. Grumet, Michigan State University, East Lansing, MI, U.S.A.

M. L. Guerinot, Dartmouth College, Hanover, NH, U.S.A.

S. J. Gurr, University of Oxford, Oxford, U.K.

D. Guttman, University of Toronto, Toronto, Ontario, Canada

R. Hamelin, Natural Resources Canada, Québec, Canada

A. R. Hardham, ANU, Canberra, Australia

G. E. Harman, Cornell University, Geneva, NY, U.S.A.
M. Harrison, Boyce Thompson Institute, Ithaca, NY, U.S.A. A. Hartmann, GSF - Research Center for Environment and

Health GmbH, Neuherberg, Germany

B. Hause, Leibniz Institute of Plant Biochemistry, Halle,

Sachsen-Anhalt, Germany

M. C. Hawes, University of Arizona, Tucson, AZ, U.S.A.

M. Hayashi, Osaka University, Suita , Osaka, Japan

S. Y. He, Michigan State University, East Lansing, MI, U.S.A.

A. Herrera-Estrella, Centro de Investigacion y de Estudios Avanzados, Irapuato Gto, Mexico

H. Hirt, Gregor Mendel-Institut, GMI GmbH, Vienna, Austria

M. Höfte, Ghent University, Gent, Belgium

E. B. Holub, University of Warwick, Wellesbourne, U.K.

B. Howlett, The University of Melbourne, Parkville,

Victoria, Australia

R. Hueckelhoven, Justus-Liebig-University Giessen,

Giessen, Germany

E. Huitema, Case Western Reserve University, Cleveland, OH, U.S.A.

S. Hulbert, Kansas State University, Manhattan, KS, U.S.A.

S. Hutcheson, University of Maryland, College Park, MD, U.S.A.

I. Hwang, Seoul National University, Seoul, Korea

R. Innes, Indiana University, Bloomington, IN, U.S.A.

M. M. Jahn, Cornell University, Ithaca, NY, U.S.A.

G. Jakab, University of Pecs, Pecs, Hungary

E. James, School of Life Sciences, University of Dundee,

Dundee, U.K.

M. Jebbar, University of Rennes 1, Rennes, France

G. Johal, Purdue University, West Lafayette, IN, U.S.A.

A. W. B. Johnston, University of East Anglia, Norwich, U.K.

D. Jones, Research School of Biological Sciences, Canberra, ACT, Australia

J. B. Jones, University of Florida, Gainesville, FL, U.S.A.

M. H. A. J. Joosten, Wageningen University, Wageningen, The Netherlands

P. Kachroo, University of Kentucky, Lexington, KY, U.S.A.

M. L. Kahn, Washington State University, Pullman, WA, U.S.A.

I. Kaloshian, University of California, Riverside, CA, U.S.A.

F. Kamilova, Leiden University, Leiden, The Netherlands

S. Kamoun, The Ohio State University, Wooster, $\mathrm{OH}$,

U.S.A.

S. Kang, Penn State University, University Park, PA, U.S.A.

E. Kannenberg, The University of Georgia, Athens, GA, U.S.A.

A. Karasev, Thomas Jefferson University, Doylestown, PA,

U.S.A.

M. Kawaguchi, University of Tokyo, Tokyo, Japan

M. Keller, Institut de Biologie Moléculaire des Plantes, UPR

CNRS 2357, Université Louis Pasteur, Strasbourg, France

B. Keller, University of Zurich, Zurich, Switzerland

G. H.J. Kema, Plant Research International, WUR,

Wageningen, The Netherlands

J. Kijne, Leiden University, Leiden, The Netherlands

H. Klee, University of Florida, Gainesville, FL, U.S.A.

J. Kniskern, University of Chicago, Chicago, IL, U.S.A

W. Knogge, Leibniz-Institute of Plant Biochemstry, Halle,

Germany

R. Koebnik, Martin-Luther-UniversitätInstitut für

GenetikWeinbergweg 10, Halle (Saale), Germany

L. M. Kohn, University of Toronto, Mississauga, Ontario, Canada 
M. V. Kolomiets, Texas A\&M University, College Station, TX, U.S.A.

H. Koltai, ARO, Volcani Center, Bet Dagan, Israel

A. Krause, University of Bremen, Bremen, Germany

H. B. Krishnan, USDA-ARS, University of Missouri,

Columbia, MO, U.S.A.

K. Kuchitsu, Tokyo University of Science, Noda, Chiba, Japan

U. Kück, Ruhr-Universität Bochum, Bochum, Germany

H. Küster, Bielefeld University, Bielefeld, Germany

M. C. Laus, Institute of Biology Leiden, Leiden, The

Netherlands

C. Lawrence, Virginia Polytechnic Institute and State

University, Blacksburg, VA, U.S.A.

J. E. Leach, Colorado State University, Fort Collins, CO, U.S.A.

V. Lefebvre, INRA, Montfavet-Cedex, France

D. LeRudulier, UMR CNRS-INRA-Universite de Nice-

Sophia Antipolis, Sophia Antipolis, France

J. Leveau, Netherlands Institute of Ecology (NIOO-KNAW),

Heteren, The Netherlands

A. Levine, The Hebrew University of Jerusalem, Jerusalem, Israel

J. Lindbo, Ohio State University, Wooster, OH, U.S.A.

S. E. Lindow, University of California, Berkeley, CA,

U.S.A.

J. Linz, Michigan State University, East Lansing, MI, U.S.A.

R. Loria, Cornell University, Ithaca, NY, U.S.A.

T. Lough, Genesis, Auckland, New Zealand

R. L. Mach, TU Wien - Institute for Biochemical

Technology and Microbiology, Vienna, Austria

M. Machado, Instituto Agronômico, Cordeiropolis, SP, Brazil

D. Mackey, Ohio State University, Columbus, OH, U.S.A.

J. Mansfield, Imperial College, Ashford, U.K.

S. Marinello, Research Institute for Industrial Crops, Bologna, Italy

F. Martin, Institut National de la Recherche Agronomique, Champenoux, France

G. B. Martin, Cornell University, Ithaca, NY, US.A.

J. L. Martinez, Centro Nacional de Biotecnologia-CSIC, Madrid, Spain

U. Mathesius, Australian National University, Canberra, Australia

F. Mauch, University of Fribourg, Fribourg, Switzerland

A. G. Maule, Queen's University Belfast, Belfast, U.K.

A. J. Maule, John Innes Centre, Norwich, U.K.

M. Megias, University of Sevilla, Sevilla, Spain

L. Mehli, Sør-Trøndelag University College, Trondheim,

Norway

P. Mergaert, CNRS, Gif-sur-Yvette, France

B. Meyers, Blake University, Newark, DE, U.S.A.

J. Michiels, Katholoieke Univ Leuven, Heverlee, Belgium

A. H., Millar, The University of Western Australia, Perth,

Australia

E. Mirkov, Texas A\&M University, Weslaco, TX, U.S.A.

T. Mitchell, North Carolina State University, Raleigh, NC, U.S.A.

B. Moerschbacher, Institut fuer Biochemie und

Biotechnologie der Pflanzen, Münster, Germany

A. Molina, Universidad Politecnica Madrid, Madrid, Spain

J. Moyer, North Carolina State University, Raleight, NC, U.S.A.

G. Muehlbauer, University of Minnesota, St. Paul, MN, U.S.A.

B. Mueller-Roeber, University of Potsdam, Potsdam- Golm, Germany
K. Mysore, Samuel Roberts Noble Foundation, Ardmore, OK, U.S.A.

U. Nehls, Eberhard-Karls-Universitaet, Tuebingen,

Germany

R. Nelson, Samuel Roberts Noble Foundation, Ardmore, OK, U.S.A.

M.-A. Newman, Royal Veterinary and Agricultural

University, Frederiksberg C, Denmark

K. Niehaus, University Bielefeld, Bielefeld, Germany

D. Noel, Marquette University, Milwaukee, WI, U.S.A.

T. Nuernberger, Eberhard-Karls Univ Tubingen, Tubingen, Germany

O. Nybroe, Royal Veterinary and Agricultural University,

Frederiksberg, Denmark

G. Oldroyd, John Innes Centre, Norwich, U.K.

J. Ollero, Universidad de Sevilla, Sevilla, Spain

R. Omarov, Texas A\&M University, College Station, TX, U.S.A.

A. Osbourn, The Sainsbury Laboratory, John Innes Centre,

Norwich, U.K.

R. Panstruga, MPI für Züchtungsforschung, Köln, Germany

E. J. Patriarca, Institute of Genetics and Biophysics A.

Buzzati-Traverso, C.N.R., Naples, Italy

J. E. Penner-Hahn, University of Michigan, Ann Arbor, MI, U.S.A.

N. Perna, University of Wisconsin, Madison, WI, U.S.A.

C. M.J. Pieterse, Utrecht University, Utrecht, The

Netherlands

K. M. Plummer, La Trobe University, Melbourne, Victoria,

Australia

B. Poinssot, Université de Bourgogne, Dijon, France

P. Poole, University of Reading, Reading, U.K.

A. L. T. Powell, University of California, Davis, CA

G. Prestion, University of Oxford, Oxford, U.K.

A. Pugin, UMR INRA - Univ De Bourgogne, Dijon, France

P. Putnoky, University of Pecs, Pecs, Hungary

J. M. Raaijmakers, Wageningen University, Wageningen,

The Netherlands

P. Rainey, University of Auckland, Auckland, New Zealand

A. F. Ram, IBL, Leiden University, Leiden, The Netherlands

R. Ranjeva, CNRS, Castanet-Tolosan, France

S. K. Rasmussen, Royal Veterinary and Agricultural

University, Frederiksberg, Denmark

D. Reinhardt, University of Fribourg, Fribourg, Switzerland

M. Rep, University of Amsterdam, Amsterdam, The

Netherlands

B. Reuhs, Purdue University, West Lafayette, IN, U.S.A.

T. Ritsema, Utrecht Unversity, Utrecht, The Netherlands

P. Rodriquez-Palenzuela, ETS Ingenieros Agronomos,

Madrid, Spain

M. Romantschuk, University of Helsinki, Lahti, Finland

P. C. Ronald, University of California, Davis, CA, U.S.A.

T. Rouxel, INRA, Versailles, France

M. San Francisco, Texas Tech, Lubbock, TX, U.S.A.

N. Sandal, University of Aarhus, Aarhus C, Denmark

U. Schaffrath, Institut fuer Biologie III, Aachen, Germany

W. L. Schneider, USDA-ARS Foreign Disease Weed

Science, Fort Detrick, MD, U.S.A.

M. Schobert, TU-Braunschweig, Braunschweig, Germany

J. Schoelz, University of Missouri, Columbia, MO, U.S.A.

A. Schouten, Wageningen University, Wageningen, The

Netherlands

A. Schulz, The Royal Veterinary and Agricultural

University, Frederiksberg C, Denmark

P. Schweizer, Institute of Plant Genetics and Crop Plant

Research (IPK), Gatersleben, Germany

J. C. Setubal, Virginia Tech, Blacksburg, VA, U.S.A. 
Y. Shachar-Hill, Michigan State University, East Lansing, MI, U.S.A.

D. Shah, Donald Danforth Plant Science Center, St Louis, MO, U.S.A.

J. Shah, Kansas State University, Manhattan, KS, U.S.A.

A. Sharon, Tel Aviv University, Tel Aviv, Israel

K. Shirasu, Sainsbury Laboratory, Norwich, U.K.

A. Skorupska, University of M. Curie-Sklodowska, Lublin, Poland

G. Smant, Wageningen University, Wageningen, The Netherlands

B. Sobral, Virginia Tech, Blacksburg, VA, U.S.A.

R. Sonti, Centre for Cellular and Molecular Biology, Hyderabad, India

H. Spaink, Leiden University, Leiden, The Netherlands

J. Staal, Swedish University of Agricultural Sciences, Uppsala, Sweden

G. Stacey, University of Missouri, Columbia, MO, U.S.A.

H. Stotz, Oregon State, Corvallis, OR, U.S.A.

J. Streeter, Ohio State University, Wooster, OH, U.S.A.

B. Surek, University De Perpignan, Perpignan, France

L. J. Szabo, USDA ARS, St. Paul, MN, U.S.A.

X. Tang, Kansas State University, Manhattan, KS, U.S.A.

J. Thaler, Cornell University, Ithaca, NY, U.S.A.

R. Thilmony, USDA-ARS-Western Regional Research

Center, Albany, CA, U.S.A.

L. S. Thomashow, USDA-ARS, Pullman, WA, U.S.A.

M. Thon, Texas A\&M University, College Station, TX,

U.S.A.

H. Thordal-Christensen, Royal Veterinary and Agricultural

University, Frederiksberg C, Denmark

A. Tomas, Pioneer Hi-Bred Int'l, Wilmington, DE, U.S.A.

J. Ton, University of Utrecht, Utrecht, The Netherlands

P. Tornero, Universidad Politécnica de Valencia, Valencia, Spain

A. Torres, University of Texas Medical Branch, Galveston, TX, U.S.A.

T. Torto-Alalibo, Virginia Polytechnic Institute and State University, Blacksburg, VA, U.S.A.

F. Trail, Michigan State University, East Lansing, MI, U.S.A.

B. Trevaskis, CSIRO, Canberra, ACT, Australia

S. Tsuyumu, Shizuoka University, Shizuoka, Japan

S. Tucker, University of Arizona, Tucson, AZ, U.S.A.

B. Tudzynski, Westfalische Wilhelms Universität, Muenster, Germany

B. G. Turgeon, Cornell University, Ithaca, NY, U.S.A.

J. Turner, University of East Anglia, Norwich, U.K.

M. S. Ullrich, International University of Bremen, Bremen, Germany

S. R. Uppalapati, Oklahoma State University, Stillwater, OK, U.S.A.
L. Vaillancourt, University of Kentucky, Lexington, KY, U.S.A.

J. P. T. Valkonen, University of Helsinki, Helsinki, Finland

M. Valls, CNRS-INRA, Toulouse, France

A. van Brussel, IBL, Leiden University, Leiden, The

Netherlands

G. Van den Ackerveken, University of Utrecht, Utrecht, The

Netherlands

E. van der Vossen, Plant Research International, WUR,

Wageningen, The Netherlands

F. Van Gijsegem, Laboratoire De Pathologie Vegetale, Paris, France

J. van Kan, Wageningen University, Wageningen, The Netherlands

M.-A. Van Sluys, University of Sao Paulo, Sao Paulo, Brazil J. Vanderleyden, Katholieke Univ Leuven, Heverlee,

Belgium

M. L. Vasil, UCDHSC - Fitzsimmons Campus, Aurora, CO, U.S.A.

D. P. Verma, Ohio State University, Columbus, OH, U.S.A.

B. A. Vinatzer, Virginia Tech, Blacksburg, VA, U.S.A.

P. Vinuesa, Centro de Ciencias Genómicas-UNAM,

Cuernavaca, Morelos, Mexico

S. von Bodman, University of Connecticut, Storrs, CT, U.S.A.

B. Wallace, University of London, London, U.K.

J. Walton, Michigan State University, East Lansing, MI, U.S.A.

G.-L. Wang, The Ohio State University, Columbus, OH,

U.S.A.

R. R. Weigel, Albrecht von Haller Institute of Plant Sciences General and Developmental Plant Physiology, Goettingen, Germany

C. Whistler, University of New Hampshire, Durham, NH, U.S.A.

C. Williams, USDA/ARS Purdue University, West

Lafayette, IN, U.S.A.

V. Williamson, University California, Davis, CA, U.S.A.

R. P. Wise, Iowa State University, Ames, IA, U.S.A.

C. Woloshuk, Purdue University, West Lafayette, IN, U.S.A.

B. B. Wulff, IBMCP, Valencia, Spain

J.-R. Xu, Purdue University, West Lafayette, IN, U.S.A.

N. Yalpani, Pioneer Hi-Bred International, Inc., Johnston,

IA, U.S.A.

Y. Yang, University of Arkansas, Fayetteville, AR, U.S.A.

C.-H. Yan, University of Wisconsin, Milwaukee, WI, U.S.A.

B. Yang, Kansas State University, Manhattan, KS, U.S.A

B. Zhao, University of California, Berkeley, CA, U.S.A.

J.-M. Zhou, Kansas State University, Manhattan, KS, U.S.A.

L. Zimmerli, University of Neuchatel, Neuchatel,

Switzerland 